\title{
Evaluation of Proficiency Test Results in Tensile Test of Elastomer Materials
}

\author{
Kemal Kuş ${ }^{1 *}$ (iD), Bülent Aydemir 2 (iD) \\ ${ }^{1}$ VESTEL Beyaz Eşya Tic.San.A.Ş., Keçili/Şehzadeler / MANISA,TÜRKIYYE \\ 2 TÜBITTAK Ulusal Metroloji Enstitüsü (TÜBITTAK UME), Gebze/Kocaeli, TÜRKIYYE \\ *kemal.kus@vestel.com.tr \\ *Orcid: 0000-0001-8189-0196
}

Received: 22 February 2021

Accepted: 6 September 2021

DOI: $10.18466 /$ cbayarfbe. 884874

\begin{abstract}
Testing laboratories need to verify their test procedures and testing capability for reliable results. In this study, equivalent samples are prepared with elastomer materials. This paper has described the evaluation of tensile test results of elastomer material specimens tested according to ISO 37 and DIN 53504 standards. The proficiency test conditions, test equipment and the results were evaluated according to TS EN ISO/IEC 17043 standard. Finally, the Z scores of the participant laboratories were presented.
\end{abstract}

Keywords: Proficiency test (PT), plastic material, tensile test, ISO 37, DIN 53504, EN ISO/IEC 17043

\section{Introduction}

General conditions for the competence of test and calibration laboratories TS EN ISO / IEC 17025 standard has been defined. It includes the evaluation, approval and subsequent inspection of the technical competence of the laboratory according to the necessary criteria by an internationally recognized and authorized organization to ensure that the tests and analyzes are carried out with TS EN ISO / IEC 17025 accreditation [1].

A proficiency test is defined as one of the accreditation requirements. Interlaboratory comparisons and proficiency tests (PT) are important tools in assessing the technical competence of calibration and experimental laboratories. This issue is specified in TS EN ISO / IEC 17025, TS EN ISO / IEC 17043 and ASTM E 1301 standards [1-3] and TURKAK document [4]. It is a requirement of TURKAK that accredited laboratories or laboratories applying for accreditation participate in the PT program and / or inter-laboratory comparisons regarding the measurement sizes within the scope of accreditation and achieve successful results.

In this study, the proficiency test was carried out in the field of tensile testing of elastomer materials in accordance with ISO 37 and DIN 53504 [5,6] standards. Participating laboratories have carried out the required experiments using their material testing machines. The measurement results were sent to the Vestel laboratory in the required format, and Vestel prepared the proficiency test report by evaluating the results from the laboratories.

In this study, the results obtained during the proficiency test were discussed, in particular, an analysis was applied in accordance with the TS EN ISO / IEC 17043 standard to evaluate the differences between the average values of test results from different laboratories.

\section{Technical Protocol}

First of all, proficiency test technical protocol was prepared and sent to all participating laboratories. The technical protocol is prepared to include the preparation of test samples, distribution to laboratories, timetable for tests, test reports and test requirements according to ILAC P9 and ISO/IEC Guide 43-2 [7,8].

\subsection{Participants}

Three different test laboratories in Turkey participated in this proficiency test. The proficiency test was started on 15.06.2020 and completed on 29.06.2020. Measurements were completed in accordance with the planned time schedule.

\subsection{Samples and Test Conditions}

The sample materials are the standard raw material used in the washing machine gaskets obtained from rubber 
known as EPDM-01 A in the market. The samples were prepared from elastomer material with the same chemical composition and dimensions in accordance with ISO 37 Type-2 and DIN 53504 S2 (Figures 1a and 1b). The samples were cut by stamping using the appropriate cutting die in the standard. 5 samples were sent to each participant by numbering appropriately. After the prepared samples are transferred to the relevant laboratory, the laboratory has been made to test as soon as possible. The parameters given in ISO 37 and DIN 53504 standards were used for the necessary conditions during the proficiency tests. In the tests, the elongation measurements were obtained from the values obtained from the moving table of the machine and the stress values were obtained by dividing the values obtained from the force measuring device on the machine by the test sample cross-sectional area. The test speed was used as $500 \mathrm{~mm} / \mathrm{min}$. The grip to grip distance was used taken $50 \mathrm{~mm}$.

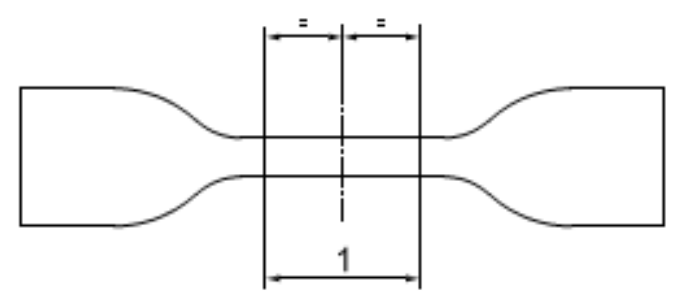

\begin{tabular}{|c|c|c|c|c|c|}
\hline Test sample type & Type & Type & Type & Type & Type \\
& 1 & $1 \mathrm{~A}$ & 2 & 3 & 4 \\
\hline Test sample & $25 \pm$ & $20 \pm$ & $20 \pm$ & $10 \pm$ & $10 \pm$ \\
length (1) (mm) & 0,5 & $0,5 *$ & 0,5 & 0,5 & 0,5 \\
\hline *The length of the test should not exceed the length of the \\
narrow part of the test piece. (Dimension of C in Table 2). \\
\hline
\end{tabular}

Figure 1a. Tensile test specimen type - Type 2 (ISO 37)

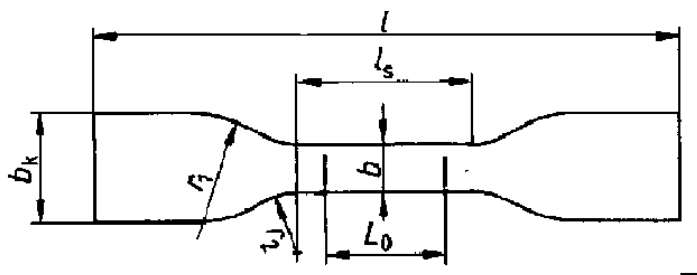

\begin{tabular}{|c|c|c|c|c|}
\hline $\begin{array}{c}\text { Test sample type } \\
\text { Dimension (mm) }\end{array}$ & S 1 & S 2 & S 3 & S 3A \\
\hline length, 1 & 115 & 75 & 35 & 50 \\
\hline $\mathrm{b}_{\mathrm{k}}$ & 25 & 12,5 & 6 & 8,5 \\
\hline $\mathrm{l}_{\mathrm{s}}$ & 33 & 25 & 12 & 16 \\
\hline $\mathrm{b}$ & 6 & 4 & 2 & 4 \\
\hline $\mathrm{r}_{1}$ & 25 & 12,5 & 3 & 10 \\
\hline $\mathrm{r}_{2}$ & 14 & 8 & 3 & 7,5 \\
\hline $\mathrm{a}$ & $2 \pm 0,2$ & $2 \pm 0,2$ & $1 \pm 0,1$ & $2 \pm 0,2$ \\
\hline $\mathrm{L}_{0}$ & 25 & 20 & 10 & 10 \\
\hline
\end{tabular}

Figure 1b. Tensile test specimen type - S2 (DIN 53504)

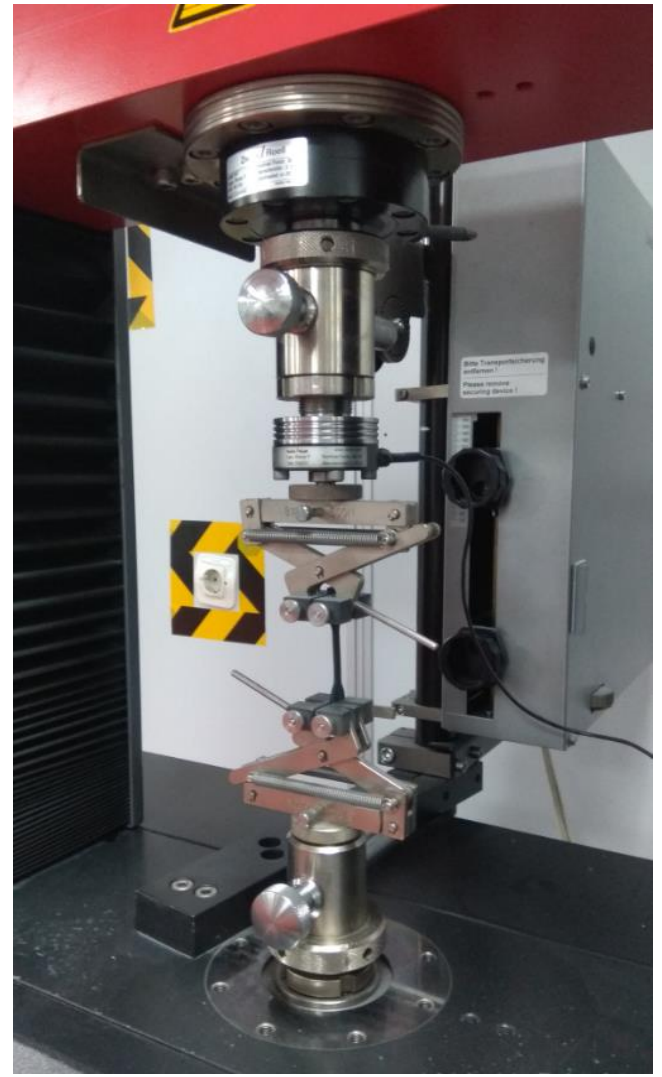

Figure. 1c. Tensile test device photo, sample, extensometer and force measuring device

Laboratories that have the infrastructure and that are or will be accredited by TURKAK participated in the proficiency test. In order to keep their identities confidential, the results are given with the lab code

\section{Results and Discussion}

After completing the tests by each laboratory, the test reports were sent to the Vestel laboratory. Vestel laboratory evaluated all test reports according to TS EN ISO / IEC 17043 and ISO 13528 [9]. standards. In given figures and tables, each laboratory is represented with a letter as $\mathrm{A}, \mathrm{B}, \mathrm{C}$. The measurement results are prepared using documents from the laboratories participating in the proficiency test and the distributions between the values declared by the participants are given in the tables below. In addition, statistical data and results were created in accordance with the requirements of the TS EN ISO / IEC 17043 standard. Homogeneity and stability tests were carried out for the samples according to ISO 5725-1, ISO 5725-2 and ISO 5725-4 [10,11,12] standarts. However, the details are not given in this study.

Measurement results of participating laboratories in accordance with the ILAC-P10 [13] policy; calculated according to the following formula specified in the standard of TS EN ISO / IEC 17043. 
$\mathrm{Z}$ score is taken as an assessment method of the evaluated results. The $\mathrm{Z}$ score is calculated according to below equation:

$$
z=\frac{x-X}{\sigma}
$$

$X \quad$ : Reference value

$X \quad$ : Participating in the laboratory result

$\sigma \quad$ : Standard deviation for proficiency testing evaluation

Criteria for performance evaluation should be established after taking into account whether the performance measure involves certain features. The features of $\mathrm{z}$ scores for performance evaluation are as follows;

$|\mathrm{z}|<2.0$ shows adequate performance and does not generate a warning signal.

$2.0<|\mathrm{z}|<3.0$ shows questionable performance and generates a warning signal, the problem should be investigated.

$|z|>3.0$ shows poor performance and corrective action should be implemented.

The robust test method was used to determine the assigned value. After the proficiency test program was completed, the robust method was applied statistically to the results obtained. While applying the method, regardless of the value, the results were not eliminated and used as they were, but the assigned value was determined by reducing the effect of the extreme values with the help of the method. The following steps were followed during the implementation process. The $p$ pieces of data (delivered results) belonging to the participants are listed in ascending order from smallest to largest as follows:

$$
x_{1}, x_{2}, \ldots, x_{i}, \ldots, x_{p-1}, x_{p}
$$

The robust mean $\left(\mathrm{x}^{*}\right)$ and robust standard deviation $\left(\mathrm{s}^{*}\right)$ premise values of these data are calculated as follows:

$x *=x i$ median (median) of the data $(\mathrm{i}=1,2, \ldots \mathrm{p})$ The median of the values

$\mathrm{s}^{*}=1,483(|x i-x *|(\mathrm{i}=1,2, \ldots \mathrm{p}))$

After these preliminary values are calculated, the robust mean $\left(\mathrm{x}^{*}\right)$ and robust standard deviation $\left(\mathrm{s}^{*}\right)$ increased values are calculated using the following formulas: $\delta=1.5 \mathrm{~s} *$ is calculated and using these values, $x i *$ values are calculated again for each $x i(i=1,2, \ldots p)$ value as follows::

$$
x_{i}^{*}=\left\{\begin{array}{cc}
x^{*}-\delta, & \text { if } x_{i}<x^{*}-\delta \\
x^{*}+\delta, & \text { if } x_{i}>x^{*}+\delta \\
x_{i}, & \text { or }
\end{array}\right.
$$

Using these new values, the new robust mean $\left(\mathrm{x}^{*}\right)$ and robust standard deviation $\left(\mathrm{s}^{*}\right)$ are calculated as follows:

$x^{*}=\sum x_{i}^{*} / p$

$s^{*}=1,134 \sqrt{\sum\left(x_{i}^{*}-x^{*}\right)^{2} /(p-1)}$

For deviating values, after making the necessary corrections according to the above evaluation process, new mean $\left(\mathrm{x}^{*}\right)$ and standard deviation $\left(\mathrm{s}^{*}\right)$ values are calculated. The $\mathrm{x}^{*}$ and $\mathrm{s}^{*}$ values are repeated until no correction is required. The correction process may need to be repeated several times. The repetition process is continued until the $3 \mathrm{rd}$ digit after the $\mathrm{x}^{*}$ and $\mathrm{s}^{*}$ comma remains constant. Thus, deviating values are corrected and included within the acceptance range. These last determined values are followed by an iterative calculation method until they converge, and the third significant figure in both values is considered to have converged as soon as there is no change from one iteration to the other. After these calculations, the last average value that is not corrected is accepted as the assigned value.

Table 1. Tensile strength values participant results

\begin{tabular}{cccccc}
\hline & \multicolumn{5}{c}{$\sigma_{\max }$ Values $(\mathrm{MPa})$} \\
\hline Lab Code & $\mathbf{1}$ & $\mathbf{2}$ & $\mathbf{3}$ & $\mathbf{4}$ & $\mathbf{5}$ \\
\hline $\mathrm{A}$ & 6,90 & 8,00 & 8,40 & 8,60 & 8,70 \\
$\mathrm{~B}$ & 8,33 & 8,17 & 8,50 & 8,95 & 9,58 \\
$\mathrm{C}$ & 8,30 & 7,50 & 7,80 & 8,10 & 8,30 \\
\hline
\end{tabular}

Table 2. Performance values of participants for tensile strength

\begin{tabular}{ccccc}
\hline \multicolumn{5}{c}{$\sigma_{\max }$ Values $(\mathrm{MPa})$} \\
\hline Lab. Code & Mean Value & $\mathbf{x}^{*}$ & $\mathbf{s}^{*}$ & Z-Score \\
\hline A & 8,12 & 8,09 & 0,283 & 0,11 \\
B & 8,71 & 0 & 9 & 2,18 \\
C & 8,00 & & & $-0,32$ \\
\hline
\end{tabular}

Table 3. Elongation values at break participant results

\begin{tabular}{cccccc}
\hline \multicolumn{7}{c}{$\varepsilon_{\mathrm{R}}$ Values $(\%)$} \\
\hline Lab Code & $\mathbf{1}$ & $\mathbf{2}$ & $\mathbf{3}$ & $\mathbf{4}$ & $\mathbf{5}$ \\
\hline A & 513 & 536 & 563 & 564 & 565 \\
B & 678 & 630 & 672 & 704 & 712 \\
C & 568 & 586 & 590 & 595 & 601
\end{tabular}


Table 4. Performance values of participants for elongation at break

\begin{tabular}{rlccc}
\hline \multicolumn{5}{c}{$\varepsilon_{\mathrm{R}}$ Values (\%) } \\
\hline Lab. & Mean & \multirow{2}{*}{$\mathbf{x}^{*}$} & $\mathbf{s}^{*}$ & Z-Score \\
Code & Value & & 52,039 & $-0,86$ \\
$\mathrm{~A}$ & 548,2 & 592,7 & & 1,66 \\
$\mathrm{~B}$ & 679,2 & & & $-0,09$ \\
$\mathrm{C}$ & 588,0 & & & \\
\hline
\end{tabular}

Table 5. 100\% modulus values participant results

\begin{tabular}{|c|c|c|c|c|c|}
\hline \multirow[b]{2}{*}{ Lab Code } & \multicolumn{5}{|c|}{$\sigma_{100}$ Values (MPa) } \\
\hline & 1 & 2 & 3 & 4 & 5 \\
\hline $\mathrm{A}$ & 2,00 & 2,00 & 2,10 & 2,10 & 2,10 \\
\hline $\mathrm{B}$ & 1,81 & 1,76 & 1,79 & 1,86 & 1,88 \\
\hline $\mathrm{C}$ & 2,30 & 2,10 & 2,10 & 2,20 & 2,20 \\
\hline
\end{tabular}

Table 6. Performance values of participants for $100 \%$ modulus

\begin{tabular}{|c|c|c|c|c|}
\hline \multicolumn{5}{|c|}{$\sigma_{100}$ Values $(\mathrm{MPa})$} \\
\hline $\begin{array}{l}\text { Lab. } \\
\text { Code }\end{array}$ & $\begin{array}{l}\text { Mean } \\
\text { Value }\end{array}$ & $\mathbf{x}^{*}$ & $\mathbf{s}^{*}$ & Z-Score \\
\hline $\mathrm{A}$ & 2,06 & 2,04 & 0,148 & 0,14 \\
\hline B & 1,82 & & & $-1,49$ \\
\hline $\mathrm{C}$ & 2,20 & & & 0,95 \\
\hline
\end{tabular}

Table 7. 300\% modulus values participant results

\begin{tabular}{cccccc}
\hline & \multicolumn{5}{c}{$\sigma_{300}$ Values $(\mathrm{MPa})$} \\
\hline Lab Code & $\mathbf{1}$ & $\mathbf{2}$ & $\mathbf{3}$ & $\mathbf{4}$ & $\mathbf{5}$ \\
\hline A & 3,60 & 3,70 & 3,70 & 3,70 & 3,70 \\
B & 3,19 & 3,07 & 3,12 & 3,18 & 3,23 \\
C & 4,00 & 3,80 & 3,80 & 3,90 & 3,90 \\
\hline
\end{tabular}

Table 8. Performance values of participants for $300 \%$ modulus

\begin{tabular}{|c|c|c|c|c|}
\hline \multicolumn{5}{|c|}{$\sigma_{300}$ Values $(\mathrm{MPa})$} \\
\hline $\begin{array}{l}\text { Lab. } \\
\text { Code }\end{array}$ & $\begin{array}{l}\text { Mean } \\
\text { Value }\end{array}$ & $\mathbf{x}^{*}$ & $\mathbf{s}^{*}$ & Z-Score \\
\hline $\mathrm{A}$ & 3,68 & 3,73 & 0,305 & $-0,16$ \\
\hline $\mathrm{B}$ & 3,16 & & & $-1,87$ \\
\hline $\mathrm{C}$ & 3,88 & & & 0,49 \\
\hline
\end{tabular}

\section{Evaluation of Performance}

Performance evaluation is based on the following performance criteria.

1) $|\mathrm{z}|<2.0$ shows adequate performance and does not generate a warning signal. $2.0<|\mathrm{z}|<3.0$ shows questionable performance and generates a warning signal, the problem should be investigated. $|\mathrm{z}|>3.0$ underperforms and generates a signal of action and corrective action should be implemented. The differences between the participants are given in the result section of the participants.

2) In the case of $Z$ Score warning signal and activity signal, possible sources of error are given below.

- the test equipment has not been calibrated.

- the test equipment is not calibrated within its operating range,

- the test equipment did not carry out intermediate checks

- Test technical person is not getting enough education

- $\quad$ used a different method as a test method.

3) If the $\mathrm{Z}$ Score value is greater than 10 , it makes it impossible to evaluate the results and interpret the performance. For this reason, participant values with a Z-Score value greater than 10 were not included in the calculations.

As a result of the evaluation of the $\mathrm{Z}$ score, the tensile strength $\left(\sigma_{\max }\right)$ value of Lab B was found to be questionable as $2<z<3$. This can often be caused by test equipment, test personnel, and test method differences. According to the results of this study, Lab B personnel should examine these parameters that affect the results in detail and determine the cause of the error. After the conditions that created the error are eliminated, Lab B should join the proficiency test again and get a successful result.

\section{Conclusion}

The laboratories, one of which is accredited by TURKAK and the other two of which will be accredited with existing infrastructure, participated in the proficiency test in order to perform the test. The experiments were carried out in accordance with the schedule. Based on the results obtained from the laboratories, this final report was prepared by the Vestel Washing Machine Incoming Quality laboratory.

As a result of the evaluation of the $\mathrm{Z}$ score, only the tensile strength $\left(\sigma_{\max }\right)$ value of the Lab B would be questionable; It has been determined that the proficiency test measurements of all laboratories in the parameters of the tensile strength $\left(\sigma_{\max }\right)$, elongation at break $\left(\varepsilon_{\mathrm{R}}\right), 100 \%$ modulus and $300 \%$ modulus are as "z $<2$ sufficient $=$ successful"

In order to verify the testing capability of the test laboratories, the proficiency tests are very important for the assessment of the tensile test measurement capabilities of all laboratories and they should be repeated periodically. 
Table 9. Overall Z score assessment

\begin{tabular}{ccccccc}
\hline & $|z|<2$ & $2<|z|<3$ & $|z| \geq 3$ & \\
\hline Z- & Num.of & \multicolumn{3}{c}{ Num.of } & Num.of & \\
Score & Lab & $\%$ & Lab & \% & Lab & $\%$ \\
\hline$\sigma_{\max }$ & 3 & 75 & 1 & 25 & & \\
$\varepsilon_{\mathrm{R}}$ & 4 & 100 & & & & \\
$\sigma_{100}$ & 4 & 100 & & & \\
$\sigma_{300}$ & 4 & 100 & & & & \\
\hline
\end{tabular}

\section{Author's Contributions}

Kemal Kuş: Drafted and wrote the manuscript, performed the experiment and result analysis.

Bulent Aydemir: Assisted in analytical analysis on the structure, supervised the experiment's progress, result interpretation and helped in manuscript preparation.

\section{Ethics}

There are no ethical issues after the publication of this manuscript.

\section{References}

1. TS EN ISO/IEC 17025, Deney ve Kalibrasyon Laboratuvarlarının Yeterliliği İçin Genel Şartlar, TSE, 2017.

2. TS EN ISO/IEC 17043: 2013, Uygunluk değerlendirmesi Yeterlilik deneyi için genel kurallar, TSE, 2017.

3. ASTM E1301, Standard Guide for Proficiency Testing by Interlaboratory Comparisons, 1995.

4. P704, TURKAK Yeterlilik Deneyleri ve Laboratuvarlararas Karşılaştırma Programları Prosedürü, Rev 10, 2019.

5. ISO 37, Rubber, vulcanized or thermoplastic - Determination of tensile stress-strain properties, International Organization for Standardization, 2017.

6. DIN 53504, Testing of rubber - Determination of tensile strength at break, tensile stress at yield, elongation at break and stress values in a tensile test, 2017.

7. ILAC P9 , Policy for Participation in Proficiency Testing Activities; 2014

8. ISO/IEC Guide 43-2 , Proficiency testing by interlaboratory comparisons - Part 2: Selection and use of proficiency testing schemes by laboratory accreditation bodies; ISO,1997.

9. ISO 13528 , Statistical methods for use in proficiency testing by interlaboratory comparison, International Organization for Standardization, 2015 .

10. ISO 5725-1; Accuracy (trueness and precision) of measurement methods and results - Part 1: General principles and definitions, 1994

11. ISO 5725-2, Accuracy (trueness and precision) of measurement methods and results - Part 2: Basic method for the determination of repeatability and reproducibility of a standard measurement method, 2019

12. ISO 5725-4, Accuracy (trueness and precision) of measurement methods and results — Part 4: Basic methods for the determination of the trueness of a standard measurement method, 2020

13. ILAC P10 , ILAC Policy on Metrological Traceability of Measurement Results ;2020. 